\title{
Ein Becher Sahne - und die Augengefäße sind gelähmt
}

\author{
Nirgends können Ärzte kleine Gefäße so gut beob- \\ achten wie im Auge. Das lässt sich zur Diagnose vas- \\ kulärer Störungen nutzen - und für aufschlussreiche \\ Experimente.
}

Trinken junge, gesunde Probanden 300 Milliliter Sahne, beeinträchtigt dies über Stunden hinweg die Endothelfunktion der retinalen Gefäße: Die neurovaskuläre Kopplung entfällt, auf Flackerlicht reagieren die Arteriolen praktisch nicht mehr. Normalerweise dehnen sich die Gefäße schnell und deutlich aus die gesteigerte Lichtverarbeitung erfordert eine erhöhte Durchblutung. Nach dem Ende des Reizes kommt es zu einer überschießenden Konstriktion, um die Durchblutung wieder zu senken. All das entfällt, wenn das Blut zu viel Lipide enthält. „Wenn Sie das nächste Mal ein Stück Torte essen, lassen Sie die Sahne besser weg, denn das Auge reagiert sehr empfindlich auf Fett", sagte Professor Tjalf Ziemssen, Zentrum für klinische Neurowissenschaften an der Universität Dresden.

\section{Prüfung mit einer Art Stroboskop}

Der Neurologe erläuterte an diesem Beispiel, welche Möglichkeiten die retinale Gefäßanalyse nicht nur bei neurologischen, sondern auch internistischen Krankheiten bietet. So können Ärzte damit mikrovaskuläre Störungen und Schäden im Auge besonders gut erkennen und auch den Therapieverlauf überprüfen. Für das Verfahren stehen heute hochauflösende Funduskameras zur Verfügung, mit denen sich der Gefäßdurchmesser bis auf die einzelne Endothelzelle bestimmen lässt. Sie ermöglichen es, in Echtzeit Veränderungen beim Durchmesser zu beobachten und den funktionellen Status der Mikrogefäße zu beurteilen. So lässt sich etwa erkennen, ob ein Schlaganfall mikro- oder makrovaskulär bedingt ist - für die Sekundärprävention ein wichtiger Unterschied ( $\bullet a b .2$ ). Die Prüfung der neurovaskulären Kopplung mit einer Art Stroboskop ist auch bei Diabetikern im frühen Krankheitsstadium oder bei Hypertonikern interessant: Hier reagieren die Gefäße ebenfalls nicht adäquat. Werden die Patienten jedoch gut eingestellt, ist oft wieder eine normale Reaktion zu beobachten. Ähnliches fällt bei Patienten mit familiärer Hypercholesterinämie nach einer Apherese auf. Charakteristische Reaktionen auf Flackerlicht sind auch bei Morbus Fabry zu sehen: Hier wird eine verstärkte Dilatation bemerkt. Ursache ist wohl eine Schädigung durch Ablagerungen des Stoffwechselprodukts Globotriaosylceramid im Endothel.

\section{Aufschlussreiche Gefäßgymnastik}

Auch die „Gefäßgymnastik“ ist dann häufig gestört. Die Patienten müssen dazu im Fünfsekundenrhythmus ein- und ausatmen. Im Idealfall oszillieren dabei auch die retinalen Gefäßdurchmesser, weil sich die Gefäße aufgrund der Blutdruckschwankungen ausdehnen und wieder zusammenziehen. Das ist häufig bei Ablagerungen in den Gefäßen nicht der Fall, so Ziemssen. Mit solchen Tests haben die Dresdener Forscher auch Schlaganfallpatienten untersucht. Lag die Ursache für den Insult in einer Mikroangiopathie, war die Gefäßdilatation deutlich gestört, weniger jedoch bei einer Makroangiopathie. Pati-

\begin{tabular}{lll} 
T2 Post-Stroke: & Retinaanalyse der Arterie & (Zwischenanalyse) \\
\hline Subgruppe & $\begin{array}{l}\text { Ausgangsdiameter } \\
\text { Vene (MW } \pm \text { SD) }\end{array}$ & $\begin{array}{l}\text { Prozentuale Dilatation } \\
\text { Vene in \% (MW } \pm \text { SD) }\end{array}$ \\
Makroangiopathie & $157,18 \pm 21,80$ & $2,79 \pm 1,93$ \\
Mikroangiopathie & $134,87 \pm 53,92$ & $3,40 \pm 2,28$ \\
Kardioembolisch & $144,80 \pm 19,76$ & $6,73 \pm 4,96$ \\
Nicht geklärt & $147,42 \pm 18,51$ & $5,15 \pm 2,66$ \\
Gesamt & $149,03 \pm 326,36$ & $3,54 \pm 2,74$ \\
Nach Vortrag Ziemssen, DGN 2016 & \\
\hline
\end{tabular}

enten mit einem kardioembolisch bedingten Verschluss von Hirngefäßen zeigten dagegen eine ganz normale Reaktion. Solche Tests könnten also helfen, die Ursachen eines Schlaganfalls besser zu ermitteln. Ziemssen wies darauf hin, dass die retinalen Gefäße sehr stark den Hirngefäßen ähnelten. Ihre Untersuchung sollte daher auch in der Neurologie einen höheren Stellenwert erhalten.

Thomas Müller, Springer Medizin

"Die Retina als diagnostisches Fenster in das Gehirn für die Neurologie. Tjalf Ziemssen: Retinale Gefäßanalyse - neueste Daten zur vaskulären Pathophysiologie", 89. DGN-Kongress, Mannheim, 21.-24.9.2016

Arbeitshilfe zur Versorgungspraxis Demenzkranker

Über schwer Demenzkranke, die sich kaum noch artikulieren und bewegen können, herrsche zum Teil noch immer die Auffassung, es seien "leere Hüllen“ oder gar "lebende Leichen", erläuterte Professor Klaus Maria Perrar vom Zentrum für Palliativmedizin der Universität Köln. Dass dies definitiv nicht der Fall ist und diese Patienten komplexe Bedürfnisse wie auch den Wunsch nach Zuwendung haben und ihren eigenen Willen ausdrücken wollen, wurde anhand eines vom Bundesforschungsministerium geförderten Projekts deutlich. Dafür wurde in der Literatur nach Erkenntnissen über die Bedürfnisse und Verhaltensweisen schwer Demenzkranker gefahndet. Da keine von insgesamt zehn geeigneten Publikationen aus Deutschland stammte, nahm man Erkenntnisse aus Feldforschungen in stationären Einrichtungen der Altenhilfe hinzu. Nachlesen lassen sich die Resultate in der rund 80-seitigen „Arbeitshilfe für die Versorgungspraxis der stationären Altenhilfe" für Mitarbeiter von Kliniken und Pflegeeinrichtungen (Download: http://palliativzentrum.uk-koeln.de/zentrum-palliativmedizin/dateien/ Arbeitshilfe_Bedrfnisse_Demenz.pdf). Der beste Leitfaden nützt jedoch nichts, wenn er in der Praxis keine Beachtung findet. Deshalb ist in einem aktuellen Folgeprojekt geplant, die Arbeitshilfe im Pflegealltag zu implementieren, ihre Praxistauglichkeit festzustellen und sie auf die ambulante Altenhilfe auszudehnen, so Perrar. Auch soll ihre Auswirkung auf die Lebensqualität von Demenzkranken und die Arbeit der Mitarbeiter in Pflegeeinrichtungen evaluiert werden. Thomas Müller, Springer Medizin

„Palliativmedizin in der Neurologie. Bedürfnisse am Lebensende von Menschen mit schwerer Demenz", 89. DGN-Kongress Mannheim, 21.-24.9.2016 